\title{
Techno-Economic Analysis of 5G Deployment Scenarios involving Massive MIMO HetNets over mmWave: A Case Study on the US State of Texas
}

\author{
Ashutosh Jha \\ Doctoral Student, Management Information Systems \\ Indian Institute of Management Calcutta \\ ashutoshj14@iimcal.ac.in
}

\author{
Debashis Saha \\ Professor, Management Information Systems \\ Indian Institute of Management Calcutta \\ ds@iimcal.ac.in
}

\begin{abstract}
The fifth generation (5G) of mobile services envisages network heterogeneity, cell densification, and high spectral efficiency using Massive MIMO, operating at millimeter-wave frequencies. Accurately assessing the potential of financial returns for such a complex network poses to operators unique challenges including techno-economic analysis leading to the identification of decision variables most sensitive to the profitability parameters. Attempting to demystify their concerns, we evaluate the profitability potential for realistic $5 G$ deployment scenarios over $28 \mathrm{GHz}$ frequency in the State of Texas. Interestingly, we discover that the total cost of ownership for $5 G$ network is about one-third of that for $4 G$ LTEAdvanced (LTE-A) deployment, yielding estimated returns amounting to $\$ 482.14$ million for the period 2020-2030. The sensitivity analyses predict profitability in $70 \%$ of the cases of $5 G$, against LTEA. For operators, the crucial levers having the maximum impact on profitability are decisions pertaining to the spectrum acquisition and the pricing of services.
\end{abstract}

\section{Introduction}

The rapidly emerging hyper-connected society is leading to tremendous growth in network connectivity, data volume, and a range of use cases. Several studies predict that the global data traffic will increase by more than 20000 times from 2010 to 2030, along with the rise in connected devices and the emergence of newer services therefrom [1]. These unprecedented growths in data traffic, connectivity and use cases call for the fifth generation $(5 \mathrm{G})$ of mobile communication systems [2]. The 5G capitalizes on its enhanced efficiencies in spectrum utilization, higher throughput per unit cost, and lower energy consumption to deliver improved user experience, apart from promising huge cost savings for operators [1],[3]. These efficiencies arise due to new air interfaces and multiple access schemes operating in high-frequency spectrum bands, such as millimeter wave (mmWave $\sim 30-300 \mathrm{GHz}$ ) [4], which allows for higher bandwidth availability (up to 1 $\mathrm{GHz}$ ) and high data rates. Countries are already in the process of allocating spectrum in frequencies, such as $28 \mathrm{GHz}$, for future $5 \mathrm{G}$ deployments [1].

New air interfaces, such as Massive MIMO, lead to very high spectral efficiency and cellular throughput when used in heterogeneous network (HetNet) scenario [1],[2]. The 5G HetNets combine several cellular layouts (Figure 1), such as macrocells, microcells, and small cells (picocells, femtocells and $\mathrm{Wi}-\mathrm{Fi}$ ), in order to achieve cooperation between lower-frequency wide-area-coverage networks and higher-frequency ultra-dense networks [3]. HetNets also allow for greater spectrum utilization (through freeing up the bandwidth via local offloading), use of unlicensed spectrum bands (Wi-Fi and femtocells) and close internetworking of communication end-points [2],[3],[4]. These advantages of HetNets enable intelligent integration of Long-Term Evolution (LTE) technologies (viz., LTE and LTE-Advanced (LTE-A)) operating in macrocellular configurations with ultradense cellular networks comprising micro and smallcells [3].

However, the real life deployment of $5 \mathrm{G}$ HetNets in mmWave band will need operators to take into account several aspects belonging to geographical characteristics of the area, demographics, future demand for services, cost of radio infrastructure, and expected revenue from services, to name a few. To address their concern, we propose a suitable technoeconomic model encompassing all the abovementioned decision variables and use the model to evaluate the achievable technical performance vis-àvis financial profitability [4],[5],[6],[7],[8],[9]. Though there are extant studies to determine the financial viability of third and fourth generations (3G and 4G) of mobile network deployments with the help of similar techno-economic models [5],[6], for the case of 
$5 \mathrm{G}$, however, there are very few studies available in the literature [4],[7],[8],[9], to the best of our knowledge. The general approach in the available literature, till now, is primarily to focus on the theoretical modeling of $5 \mathrm{G}$ cost parameters using fictional deployment scenarios [6]-[10], without delving into the revenue and profitability aspects. These studies also restrict themselves to only evaluating the impact of infrastructure costs involved in procuring and installing the network equipment and backhaul mainly [6]-[10]. The previous studies on 5G have also not considered the important decision variables of radio spectrum bandwidth and carrier frequency, which are crucial for modeling the technical performance and estimating the capital expenditure (Capex) incurred by the operators. Another aspect missing in these studies is the sensitivity analysis of the decision variables on the overall profitability indicators, such as the net present value (NPV) [11]. Though one study has performed the sensitivity analysis, it is restricted to only the fixed and variable cost components of the total cost of ownership (TCO) [11], whereas potentially such analyses for a given range of input parameter values can reveal the boundaries between which the variables of interest may lie, and also highlight the most influential input parameters in the model [12].

This paper is an attempt at furthering the research related to analyzing the techno-financial feasibility of $5 \mathrm{G}$ network deployments in the light of the abovementioned limitations. Firstly, we choose a real-life case of the state of Texas in the USA for deploying $5 \mathrm{G}$ HetNets in order to accurately model the variations in geographical characteristics and the demography. With the help of micro-level data of the county-wise distribution of population and details on the size of their land and water areas, we perform a K-Means clustering analysis [13] to segregate the areas suitable for $5 \mathrm{G}$ HetNet deployment. This renders our study less fictitious. Though we have used the Texas state data in our analysis, our methodology is generic enough to be applied to any geographical region anywhere in the world. Secondly, to better reflect the influence of spectrum considerations in the cost modeling, we fix $28 \mathrm{GHz}$ as an example carrier frequency [2] that operates under different network configurations and cellular layouts in those clusters [14]. In this context, we remind the readers that the $5 \mathrm{G}$ cellular systems are likely to operate in or near the mmWave spectrum bands of $30-300 \mathrm{GHz}$ owing to the availability of massive amount of bandwidth for both cellular and backhaul services in these bands [1],[15]. We have chosen $28 \mathrm{GHz}$ frequency band for our analysis considering several ongoing trials in that band, which are shaping up to be positive for real-life deployment

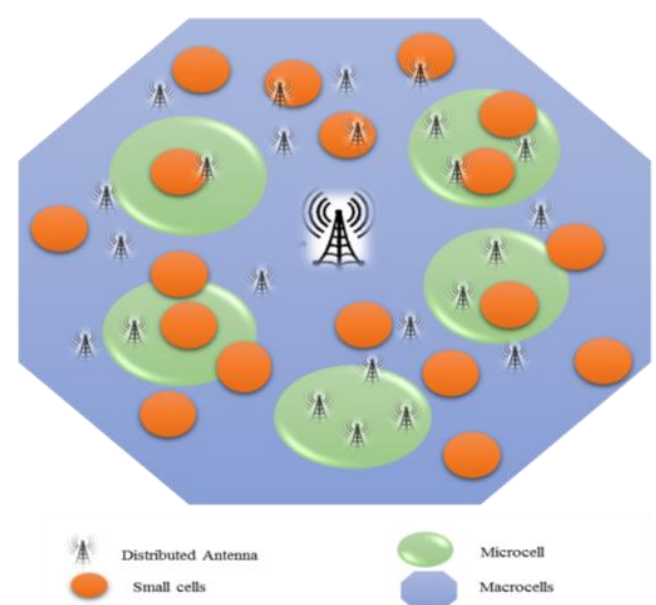

Figure 1. 5G Heterogeneous networks

and usage [2],[3]. We choose realistic spectrum block sizes of $500 \mathrm{MHz}$ for $5 \mathrm{G}$. For comparative analysis with LTE-Advanced (LTE-A) networks, we take 700 $\mathrm{MHz}$ frequency band with a block size of $10 \mathrm{MHz}$ typical for 4G [1],[6]. Thirdly, to obtain a close estimation of future subscription of $5 \mathrm{G}$ services after its launch (say in the year 2020), we take help of the Simple-Logistic growth model [16], widely used for forecasting the growth in the market size of newer products and innovations. These forecasts also help us estimate revenue from $5 \mathrm{G}$ subscribers in the plan period. Fourthly, and finally, we conduct a sensitivity analysis through a range of input values for the decision variables in our techno-economic model. The analysis generates the overall possible variation in the final profitability indicators and identifies the most significant decision variables in the model for the possible consumption by the potential operators in Texas.

The remainder of this paper is structured as follows. Section 2 provides an overview of the related literature and therefrom draws upon our research objectives. In Section 3, we explain our research methodology, providing details on various components of our techno-economic model along with their theoretical background. In Section 4, we provide the dataset used, the input values of the decision variables, and the subsequent results. Finally, Section 5 concludes on our findings and identifies the implications for praxis.

\section{Related Works and Motivation}

This section provides a brief overview of previous publications (Table 1) that have studied the technoeconomics behind provisioning of $5 \mathrm{G}$ wireless networks under different deployment scenarios. In 
Table 1. Summary of selected works on techno-economic evaluations of $5 \mathrm{G}$ networks

\begin{tabular}{|l|l|l|l|}
\hline Paper & Deployment Scenario & Technology & Model Parameters \\
\hline$[10]$ & Microcells, Picocells and Femtocells & mmWave & Capex and Opex \\
\hline$[7]$ & Macrocells & SDN, NFV & Capex, Opex and TCO \\
\hline$[8],[11],[18]$ & Picocells and Femtocells & Ultra-Dense, DAS & Capex, Opex and TCO \\
\hline$[19]$ & Macrocells, Picocells and Femtocells & 5G RAN & Capex, Opex and TCO \\
\hline$[17]$ & $\begin{array}{l}\text { Macrocells, Microcells, Femtocells, } \\
\text { and Macro-Femto HetNet }\end{array}$ & 5G RAN & Capex and Opex \\
\hline$[4]$ & Metrocells and Microcells & mmWave & Capex, Opex, Revenue and EBIT \\
\hline$[9]$ & $\begin{array}{l}\text { Macrocells, Microcells, Picocells and } \\
\text { Femtocells }\end{array}$ & LTE, LTE-A & Capex and Opex \\
\hline
\end{tabular}

addition, we introduce related literature coherent with various modeling approaches undertaken in this study. We then summarize the research gaps and propose our extensions.

\subsection{Techno-Economics of $5 G$ Networks}

In one of the early works [4], authors propose a brief conceptual model for cost calculations in $5 \mathrm{G}$ network deployments, keeping in mind the capacity aspect of the network mainly. Through a fictional deployment scenario in an urban setting with varying levels of assumed demand, their study attempts to evaluate the profit margins (via EBIT) [4]. Their study also compares, in terms of returns, 5G network deployments with 4G LTE-A deployments [4]. Another study by them [9] presents the cost-effective deployment strategies for heterogeneous wireless networks. This study evaluates the discounted costs under different combination of cellular layouts and technologies (LTE and LTE-A), assuming varying scenarios for data volume demand [9]. The study has taken into account the size of the spectrum bandwidth, albeit the choice of spectrum bands belongs to 4G LTE and LTE-A technologies only [9].

The study in [17] analyzes the technical and cost parameters of the three main technologies belonging to the network densification paradigm, namely macrocells, microcells and femtocells. The study also evaluates the cellular coverage, interference characteristics, area spectral efficiency, and energy and cost efficiency parameters under the four deployment strategies: macrocells only, microcells only, femtocells only, and macro-femto HetNets [17].

There are a couple of studies to evaluate the cost implications of integrating two enabling paradigms viz. Software Defined Networking (SDN) and Network Function Virtualization (NFV) in 5G [7]. For instance, the work in [7] compares the cost aspects (Capex, Opex and TCO) for such virtualized 5G networks vis-à-vis traditional $5 \mathrm{G}$ network. In their other works [8],[11],[18], the same authors evaluate the cost aspects (Capex, Opex and TCO) of 5G deployment scenarios involving two network architectures, namely Ultra-dense technology (femtocells) and Distributed Antenna Systems (DAS). This work also highlights the advantages of these two network architectures designed mainly to cater to the challenges of limited connectivity in indoor environments [8]. In their another work [19], they assess the financial implications of cellular network deployment using small cell technology for connectivity in indoor environments to highlight the benefits of such technology toward enhancing the cell coverage and network capacity in 5G [19]. This paper too analyzes the cost aspects (Capex, Opex and TCO) of small cells deployment from the point of view of telecom operators and also lists the subscriber incentives for choosing small cells over other access types such as WiFi [19]. The results of the technoeconomic analysis for small cells have also been compared against a macrocellular deployment scenario in order to highlight its advantages [19].

One of the recent works [10] analyzes the demand aspects of a newly proposed pricing model of $5 \mathrm{G}$ mobile services for the telecom operators in China. The work formulates the likely migration scenario of subscribers from the $4 \mathrm{G}$ mobile services to $5 \mathrm{G}$ mobile services and evaluates the sensitivities of data volume demand with respect to the price of the $5 \mathrm{G}$ mobile services [10].

\subsection{Our Research Objectives}

As evident in the previous sub-section, there are several noticeable gaps in the currently available literature. Firstly, these works do not consider any real life 5G deployment scenarios accounting for the geographical characteristics and demographic aspects of the region. Secondly, none of the above-mentioned works evaluate the future diffusion aspects of the technology and, hence, do not perform rigorous 
forecasting of the future demand of data volume. Thirdly, all the previous analyses restrict themselves to estimating TCO in radio access network (RAN) infrastructure, without considering the costs incurred in acquiring the radio spectrum which is a costly resource for the operators. The analyses also do not delve into the projected revenue from services and the overall profitability aspects of the investments. Fourthly, the sensitivities of the cost and profitability parameters with the individual decision variables in the techno-economic model have not been extensively explored in prior works. To fill in the above gaps, our work has attempted to formulate an overarching model for the techno-economic evaluation of the deployment of 5G Wireless Heterogeneous Networks (HetNets) in mmWave, which could be applied to most of the $5 \mathrm{G}$ deployment scenarios.

\section{Research Methodology}

We explain in detail the theoretical background of the steps involved in our techno-economic evaluation under three major sequences: cost modeling, revenue modeling and sensitivity analysis (Figure 2). We describe them below.

\subsection{Cost Modeling}

As already mentioned in the introductory section, we have chosen Texas State in the US as our sample case, primarily due to the availability of micro-level data on the demographics and geological characteristics of the state, and the presence of a diverse set of metropolitan, urban and rural segments with varying land areas and population densities [13]. These factors pose unique challenges to a designer in terms of estimating $5 \mathrm{G}$ usage patterns and service demands [1]. In order to segregate the land areas with similar population densities, we perform K-Means clustering, based on the geographical and population dataset of Texas. We then decide upon the type of 5G network deployment suitable to each cluster in terms of geospatial features and service usage patterns. We have considered two different $5 \mathrm{G}$ deployment scenarios to choose from, namely Dense Urban and Urban Macro, both of which belong to a larger set of deployment scenarios detailed in the $3^{\text {rd }}$ Generation Partnership Project (3GPP) specifications [1],[20].

Dense Urban HetNets combine macro and micro cells to cater for high user densities and traffic loads in city centers and dense urban areas [2]. We club the Indoor Hotspots scenario - which focuses on small coverage per cell and high user density in buildings with the Dense Urban scenario to incorporate pico and

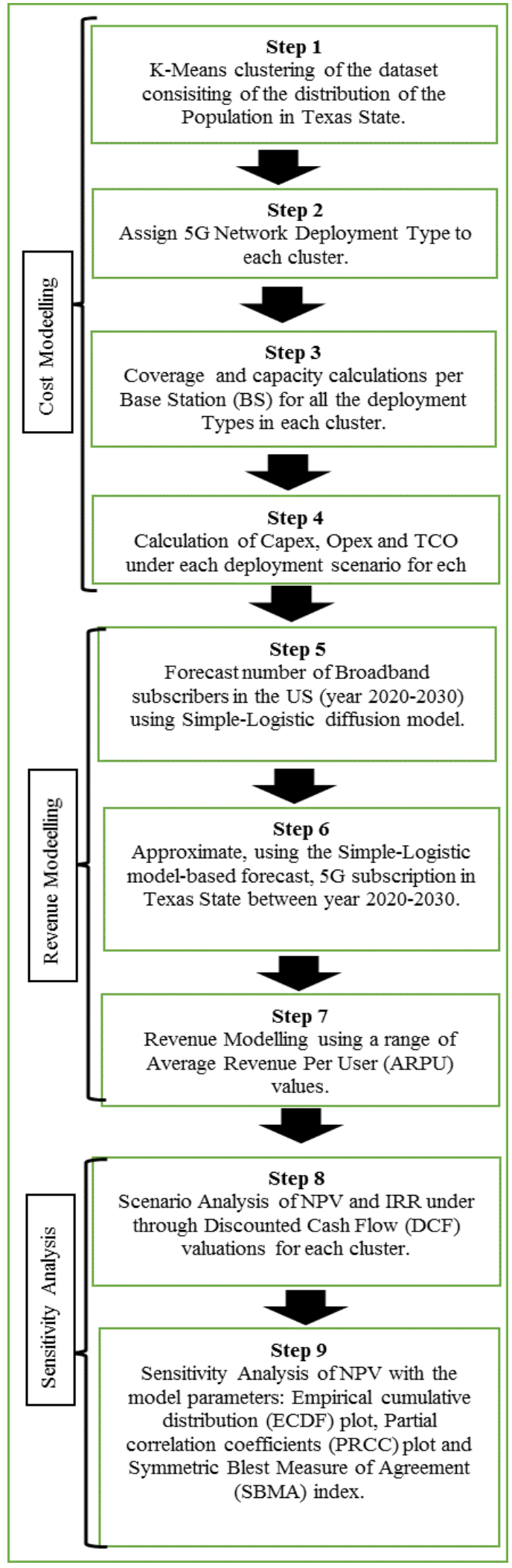

Figure 2. Steps in techno-economic analysis 
femto cellular layouts in our model. The Urban Macro scenario, on the other hand, focuses on large cells and continuous coverage in the urban and sub urban regions [1].

Our next step is to determine the achievable cellular coverage area per Base Station (BS) under each HetNet. Assuming a circular cell with radius $r_{\text {cell }}$ for the chosen frequency band, the coverage area $A_{\text {cell }}$ for each deployment scenario is simply:

$$
A_{\text {cell }}=\pi \times r_{\text {cell }}^{2}
$$

We use the cell radius values for $28 \mathrm{GHz}$ band, as obtained by Sulyman in the real-life experiments conducted at New York City and Austin, Texas, respectively [15]. Next, we use $A_{\text {cell }}$ for calculating the number of BSs required in each geographical cluster as in [9]:

$$
B S_{\text {Cluster }, i}=\left(\sum A_{\text {Cluster }, i}\right) /_{A_{\text {cell }}}
$$

where $B S_{\text {Cluster }, i}$ is the number of BSs required in the $i$-th cluster $(i \neq 0)$ and $A_{\text {Cluster }, i}$ is the land area of the $i$-th cluster. However, these many BSs may not always be sufficient to meet the aggregate data demand. We, therefore, also evaluate the achievable cellular capacity $C A P_{H N}$ in a HetNet by taking into account the allocated spectrum bandwidth (B) and the spectral efficiency $(\eta)$ of the $28 \mathrm{GHz}$ mmWave band as in [9]:

$$
C A P_{H N}=\eta \times B
$$

Taking into account the data volume demand in a cluster $\left(D_{\text {Cluster }, i}\right)$ and the average busy hour $(B H)$ traffic, we model the number of BSs required per cluster $\left(B S_{C a p, i}\right)$ as follows:

$$
B S_{\text {Cap }, i}=\left(\frac{D_{\text {Cluster } i}}{C A P_{H N} \times B H}\right)
$$

To arrive at the actual number of BSs $\left(B S_{\text {Max }, i}\right)$ which can provide full coverage as well as deliver the required capacity, we choose the maximum of $B S_{\text {Cluster }, i}$ and $B S_{\text {Cap }, i}$, i.e., $B S_{\text {Max }, i}=\max \left\{B S_{\text {Cluster }, i}\right.$ , $\left.B S_{\text {Cap }, i}\right\}$.

As we all know, the total cost of ownership (TCO) for an operator comprises two main components, Capex and Opex. Capex is the fixed cost component representing the capital investments made in RAN infrastructure (including backhaul, procurement and installation costs) and spectrum acquisition (radio spectrum is a long-lived asset). Opex, on the other hand, is the variable cost component representing the recurring costs in the operational and maintenance $\left(O A M_{\text {Cost }}\right)$ activities, site rental, energy consumption, personnel and marketing and advertising $(M A)$. Thus,

$$
\begin{gathered}
\text { Capex }_{\text {Total }}=B S_{\text {Cost }}+B S_{\text {INST }} \\
+B H L+S P_{\text {license }} \\
\text { Opex }_{\text {annual }}=O A M_{\text {Cost }}+M A
\end{gathered}
$$

where Capex $_{\text {Total }}$ is the required capital expenditure (after taking into account the total duration of the project as well as the license period of spectrum), $B S_{\text {Cost }}$ and $B S_{I N S T}$ are the costs incurred in BS equipment procurement and installation, respectively, $B H L$ is backhaul cost, $S P_{\text {license }}$ is the net cost for acquiring the block of spectrum, and Opex annual is the recurring annual expense. We assume that Capex $_{\text {Total }}$ is obtained by the operator through an infrastructure financing agreement, and the loan amount needs to be paid through a series of annual installments. For a periodic interest rate $r$ with $n$ number of repayments, the annual component of Capex $_{\text {Total }}$ is given by [8]:

$$
\text { Capex }_{\text {Annual }}=\text { Capex }_{\text {Total }}\left(\frac{r}{1-(1+r)^{-n}}\right)
$$

Then the annual TCO (TCO annual $)$ incurred by the telecom operator becomes:

$$
T C O_{\text {annual }}=\text { Capex }_{\text {Annual }}+\text { Opex }_{\text {annual }}
$$

\subsection{Revenue Modeling}

A major concern of operators while launching 5G service will be about its future adoption in different markets. To estimate future demands of $5 \mathrm{G}$, we take help of the diffusion of innovations (DOI) paradigm [16] because the diffusion of $2 \mathrm{G}, 3 \mathrm{G}$ and $4 \mathrm{G}$ services has already utilized the DOI models of Bass, Gompertz and Simple-Logistic. We use the SimpleLogistic model in this work, considering that several studies recommend it for its suitability in obtaining realistic forecasts of an innovation demand [5],[6],[16]. Simple-Logistic model is given as:

$$
\frac{d F(t)}{d t}=b_{1}\left(1-\frac{F(t)}{K}\right)
$$

where $F(t)$ is the number of subscribers at time $t, b_{1}$ is the intrinsic growth rate, and $K$ is the number of subscribers in equilibrium (i.e., ultimate market potential). The solution of (9) is as follows:

$$
F(t)=\left[\frac{K}{1+e^{-b_{1}\left(t-b_{2}\right)}}\right]
$$


where $b_{2}$ is the time offset parameter [16]. In order to forecast the future adoption of $5 \mathrm{G}$ services in the Texas State, we first need to evaluate the model parameters $\left(K, b_{1}\right.$, and $\left.b_{2}\right)$. The parameter estimation of the Simple-Logistic model requires a non-linear least squares (NLS) based regression analysis [16] using the historical data of adoption of the innovation (5G in this case).

Considering that $5 \mathrm{G}$ services will be launched in future (circa 2020), we take Broadband adoption (per 100 population) in the US as its proxy owing to the higher data rates and volume consumption patterns of Broadband, which are likely to be similar/higher in $5 \mathrm{G}$ services [1],[2],[3],[21]. First, we use the historical data of Broadband adoption to approximate the SimpleLogistic model parameters for Broadband adoption through NLS technique [16]. Then, we forecast the Broadband adoption for the decade 2020-2030 with the help of those estimated parameters [5]. This trend is then used as an indicator of the demand for $5 \mathrm{G}$ services in the Texas State. Combining the $5 \mathrm{G}$ adoption forecast $\left(N_{\text {Subsc,year }}\right)$ and the cluster-wise Average Revenue per User $\left(A R P U_{\text {Cluster }, i}\right)$, we evaluate the estimated overall annual revenue $\left(R_{\text {annual,year }}\right)$ from $5 \mathrm{G}$ services in each cluster as:

$$
R_{\text {annual,year }}=A R P U_{\text {Cluster }, i} \times N_{\text {Subsc,year }}
$$

\subsection{DCF and Sensitivity Analysis}

Harnessing Discounted Cash Flow (DCF) technique to arrive at profitability parameters of Net Present Value (NPV) and Modified Internal Rate of Return (MIRR) [22] is common in evaluating the return on investments of such a capital-intensive project as 5G deployment. A positive NPV signals a profitable scenario and vice-versa. MIRR evaluation treats positive cash flows as reinvestment options at the cost-of-capital, whereas the initial outlays are financed at the financing cost. The standard methods to calculate NPV and MIRR are as below:

$$
\begin{gathered}
N P V=\sum \frac{\left(C_{\text {Annual }}\right)}{(1+r)^{n}}-\text { Capex }_{\text {Total }} \\
M I R R=\left[\sqrt[n]{\frac{F V(\text { Positive Cash Flows, } \text { WACC })}{P V(\text { Initial Outlays, } r)}}-1\right]
\end{gathered}
$$

where $\left(C F_{\text {Annual }}\right)$ is the annual cash flow from operations (determined using (8) and (11)), $F V$ is the future value of positive cash flows at the weighted average cost of capital (WACC) for the operator, $P V$ is the present value of negative cash flows at the financing cost $(r)$ of the operator and $n$ is the number of repayments as mentioned before. We employ the
NPV and MIRR models to conduct a sensitivity analysis for the decision variables belonging to the revenue and cost models [11],[12]. Operators perform sensitivity analysis to study of variations in outputs for given uncertainties in the input parameters. We determine the empirical cumulative distribution (ECDF) of NPV/MIRR with the help of our sensitivity analysis [12]. The ECDF analysis helps operators in evaluating the implications of cost and revenue model parameters on the profitability aspects of the considered deployment scenarios [12].

\section{Evaluation Results}

We have taken the demographic and geographical dataset of Texas State in the USA from the open data source portal of the Institute for Demographic and Social Research (IDSER) [13]. The data contains the population distribution details for 254 counties of the Texas state and their corresponding land and water areas [13]. We use R language for all our modeling and computations [12].

\subsection{Cost Modeling Results}

In $\mathrm{K}$-Means clustering, we choose $\mathrm{K}=4$ to segregate the counties with similar population density into four different clusters, namely $\mathrm{C} 1$ through $\mathrm{C} 4$ (Table 2). The choice of $\mathrm{K}=4$ enables clear segregation of major cities, metropolitan areas, towns and the rural areas of the State. We can infer from Table 2 and Figure 3 that, out of 254 counties, 3 counties (under cluster C3) have extremely high population densities - signifying the presence of major cities, whereas 19 counties (under C1) are very sparsely populated - signifying the presence of nonurban areas. The remaining counties (under C2 and C4) have moderate population densities - implying metro and urban areas, respectively. We allocate the

\section{Table 2. Result of K-Means clustering}

\begin{tabular}{|l|l|l|l|}
\hline Cluster & Counties & $\begin{array}{l}\text { Pop. Density } \\
\text { (/sq. miles) }\end{array}$ & $\begin{array}{l}\text { Land Area } \\
\text { (sq. miles) }\end{array}$ \\
\hline C1 & 19 & 29.65 & 22949.3 \\
\hline C2 & 224 & 305.34 & 230761.1 \\
\hline C3 & 3 & 2405.03 & 3438.4 \\
\hline C4 & 8 & 869.80 & 6329.2 \\
\hline
\end{tabular}

deployment scenario Urban Macro (with LTE-A $\mathrm{RAN}$ ) to $\mathrm{C} 1$ and $\mathrm{C} 2$, and the deployment scenario Dense Urban (with mmWave based Massive-MIMO $\mathrm{RAN}$ ) to $\mathrm{C} 3$ and $\mathrm{C} 4$ [1]. Both scenarios include HetNets with respective specifications related to the cellular coverage, capacity and spectrum requirements 
[1],[8],[19]. Also, the design of each scenario is such that it caters to the key aspects of $5 \mathrm{G}$ experience [1].

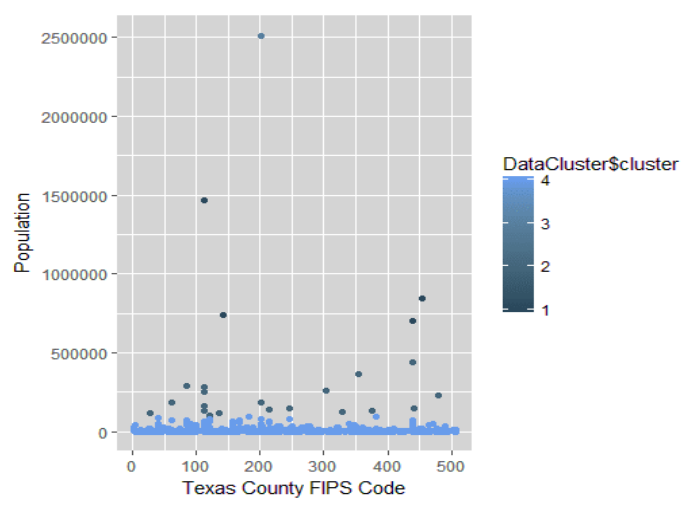

Figure 3. K-Means cluster analysis

Next, we forecast the data volume demand between years 2020-2030 for each cluster and calculate $B S_{\text {Max }, i}$ for each deployment scenario (Figure 4) using (1) (4). Since $B S_{C o v, i}$ comes out to be less than $B S_{C a p, i}$ in every case, $B S_{\operatorname{Max}, i}=B S_{C a p, i}$. Table 3 summarizes the assumptions related to the input values of carrier frequency (CF), bandwidth, cell range, spectral efficiency, and various components of Capex and Opex, along with their referential sources.

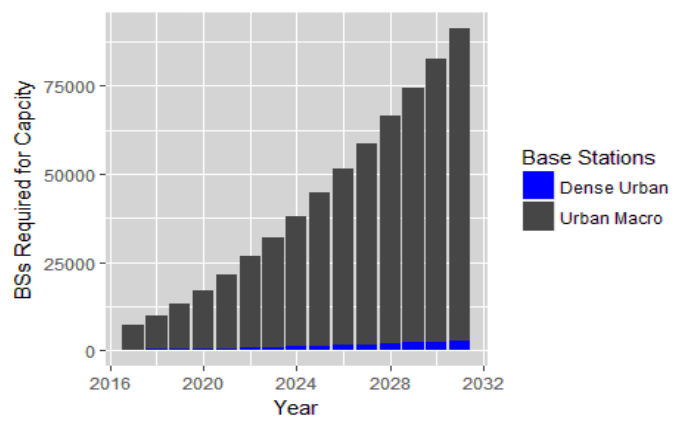

Figure 4. BSs required for capacity

As expected, Figure 4 emphasizes that much less number of BSs are required in the Dense Urban scenario as compared to the Urban Macro scenario. This is due to the higher data throughput achievable per HetNet in the Dense Urban scenario. We note here that, apart from the infrastructure costs related to the procurement and installation of a macro BS and backhaul in a $5 \mathrm{G}$ HetNet, the operator does not bear any further investments [11],[4],[19]. The subscriber needs to bear the costs for the devices pertaining to small cells (pico and femtocells), which are installed in her premises [11],[4],[19]. In our calculations, the input values for costs per macro BS in the Dense
Urban scenario takes into account the aggregate expenditure incurred in a $5 \mathrm{G}$ HetNet excluding the small-cells equipment. Thus, we may infer from the above analysis that mmWave-based Massive MIMO configurations in the Dense Urban scenario are capable of handling large data volume demands with much lower per unit infrastructure requirements as compared to Urban Macro scenario with LTE-A configurations running over $700 \mathrm{MHz}$ carrier frequencies.

We now evaluate, with the help of the input values in Table 3 and (5) - (7), the Capex, Opex and TCO required per year for each deployment scenario. We have assumed $r$ to be 0.06 per annum with $n$ equal to 10 (years) and the sector specific WACC to be at 0.07 [23], for all our cost modeling calculations. Figure 5 highlights the result of the Opex calculations under both deployment scenarios. The estimate of total Capex required amounts to $\$ 775.02$ million and \$2516.45 million, for Dense Urban and Urban Macro deployment scenarios, respectively.

Table 3. Cost modeling parameters

\begin{tabular}{|l|l|l|}
\hline Clusters & C1 + C2 & C3 + C4 \\
\hline Deployment & Urban Macro [8] & $\begin{array}{l}\text { Dense } \\
\text { Urban [8] }\end{array}$ \\
\hline RAN & LTE-A & $\begin{array}{l}\text { mmWave } \\
\text { with Massive } \\
\text { MIMO }\end{array}$ \\
\hline Frequency $(\mathrm{GHz})$ & $0.700[6]$ & $28[8]$ \\
\hline$B(\mathrm{MHz})$ & $20[6]$ & $500[8]$ \\
\hline$r_{\text {cell }}(\mathrm{km})$ & $1.25[6]$ & $0.000149[9]$ \\
\hline$A_{\text {cell }}(\mathrm{sq} . \mathrm{km})$ & $4.906[6]$ & $0.000069[9]$ \\
\hline$B S_{\text {Cov, } i}$ & 525485 & 169721982 \\
\hline$\eta(\mathrm{bit} / \mathrm{s} / \mathrm{Hz} / \mathrm{BS})$ & $3.8[6]$ & $52[24]$ \\
\hline$C A P_{H N}$ & $76 \mathrm{Mbps}$ & 26 Gbps \\
\hline$B S_{\text {Cost }}(\$)$ & $12000[6]$ & $106395[9]$ \\
\hline$B S_{\text {INST }}(\$)$ & $500[6]$ & $500[18]$ \\
\hline$B H L(\$)$ & $5000[18]$ & $5000[18]$ \\
\hline$S P_{\text {license }}(\$ / \mathrm{MHz})$ & $46000000[25]$ & $1000000[18]$ \\
\hline$O A M_{\text {Cost }}(\$ / \mathrm{year})$ & $25000[18]$ & $25000[18]$ \\
\hline
\end{tabular}

Note: These values are representative only and do not relate to any commercial product.

We observe from Figure 5 that the Opex values for the Dense Urban scenario are almost one-third of that of Urban Macro scenario. The Dense Urban HetNets have very high-cost estimates for their Massive MIMO based RAN infrastructure, due to which the Opex incurred per unit infrastructure is higher in the Dense Urban scenario as compared to the Urban Macro 
scenario. The Capex evaluations follow a trend similar to the Opex, with much higher Capex requirements in Urban Macro scenario (almost 3 times) as compared to the Dense Urban scenario. We evaluate the TCO using (8), taking into account the Capex and Opex values obtained earlier. Figure 6 highlights the results of the TCO calculations for both the deployment scenarios. Expectedly, we find the TCO values to be much higher for the Urban Macro scenario (almost 3 times) when compared to the Dense Urban scenario.

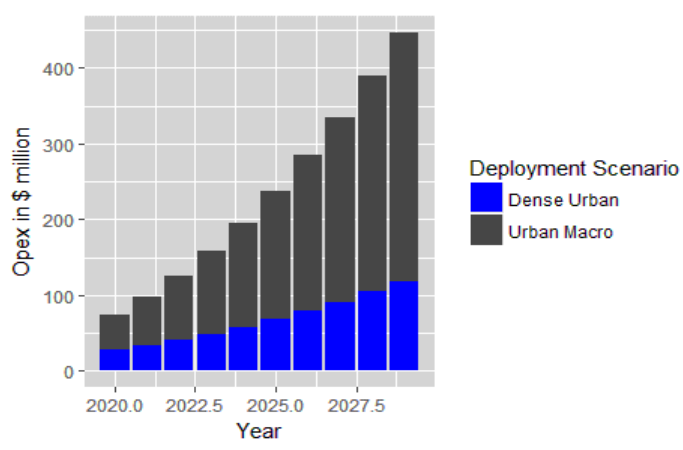

Figure 5. Opex in network deployments

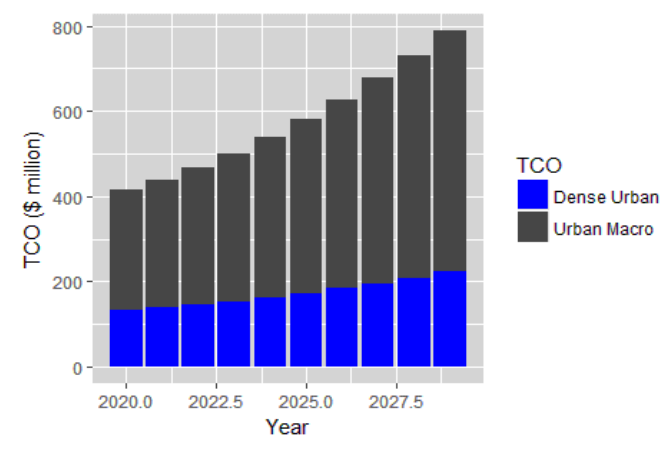

Figure 6. TCO in network deployments

\subsection{Revenue Modeling Results}

We start the revenue modeling calculations with the forecasting estimates of the number of active $5 \mathrm{G}$ subscribers in the Texas State. Table 4 summarizes the results of the NLS estimation of the Simple-Logistic model parameters using (10). Figure 7 presents the results of the $5 \mathrm{G}$ adoption forecast, which is mildly conservative, considering that the upper limit of

Table 4. NLS estimation results

\begin{tabular}{|l|l|l|l|l|}
\hline $\begin{array}{l}\text { Adj. } \\
\mathrm{R}^{2}\end{array}$ & RMSE & b1 & b2 & b3 \\
\hline 0.998 & 0.920 & $35.576^{\star \star \star}$ & $0.291^{\star \star \star}$ & $3.770^{\star \star \star}$ \\
\hline
\end{tabular}

Note: $* * *$ represents $1 \%$ level of significance subscription stagnates at 36 subscribers per 100 population. In real life deployments, however, the subscription may vary depending on the demographic patterns of the chosen cluster. In this study, we use the above forecast for modeling the revenue for both deployment scenarios. Not only we assume realistic ARPU values [21] but also we evaluate the implications of a range of ARPU values on profitability (Section 4.3).

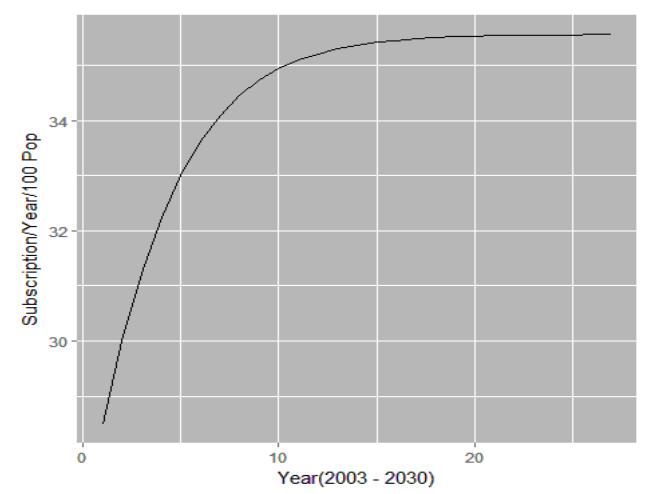

Figure 7. Forecast of $5 \mathrm{G}$ subscribers

\subsection{DCF and Sensitivity Analysis Results}

In the DCF evaluation, we generate the likely future cash flows under each deployment scenario, taking into account the individual TCO and Revenue estimates over the years. We calculate the NPV and MIRR (Table 5) of the project under each deployment scenario using (12) and (13). We find from Table 5 that Urban Macro deployment scenario indicates negative NPV, implying losses. For the Dense Urban scenario, however, the NPV is quite high, signaling high returns. We must note here that, with further maturation in the 5G RAN technologies - cost

Table 5. DCF valuation results

\begin{tabular}{|l|l|c|}
\hline & NPV (\$ million) & MIRR (\%) \\
\hline Urban Macro & -2566.496 & $-12 \%$ \\
\hline Dense Urban & 482.147 & $11 \%$ \\
\hline
\end{tabular}

effective cellular layouts in mmWave-based Massive MIMO [1], growth in compatible handheld devices, and innovative models of active and passive infrastructure sharing [3], the input values of several costs and revenue model parameters will change toward even better results. For example, the spectrum acquisition costs, RAN infrastructure costs and the ARPU values may all be different at the time of deployment of $5 \mathrm{G}$. Therefore, it becomes pertinent to evaluate the implications of these variations on the profitability aspects of deployment scenarios. So, we 
have conducted a sensitivity analysis on the NPV results, taking a wider range of input values of each decision variable (Table 6), under both deployment scenarios.

Table 6. Sensitivity analysis

\begin{tabular}{|l|l|l|}
\hline Parameters & $\begin{array}{l}\text { Urban } \\
\text { Macro }\end{array}$ & Dense Urban \\
\hline Cell range (km) & $0.65-1.55$ & $\begin{array}{l}0.000149- \\
0.000250\end{array}$ \\
\hline Bandwidth (MHz) & $10-20$ & $500-1000$ \\
\hline $\begin{array}{l}\text { Spectral Efficiency } \\
\text { (bit/s/Hz) }\end{array}$ & $2.3-5.3$ & $26-52$ \\
\hline Usage Hours & $3-13$ & $3-13$ \\
\hline Market Share (\%) & $50-100$ & $50-100$ \\
\hline ARPU (\$) & $25-95$ & $25-95$ \\
\hline $\begin{array}{l}\text { Spectrum Costs } \\
\text { \$million/MHz) }\end{array}$ & $10-50$ & $0.5-1.5$ \\
\hline BS Cost (\$) & $8000-$ & $\begin{array}{l}80000- \\
160000\end{array}$ \\
\hline Loan rate (\%) & $5-16$ & $5-15$ \\
\hline WACC (\%) & $5-15$ & $5-15$ \\
\hline Savings rate (\%) & $5-15$ & $5-15$ \\
\hline
\end{tabular}

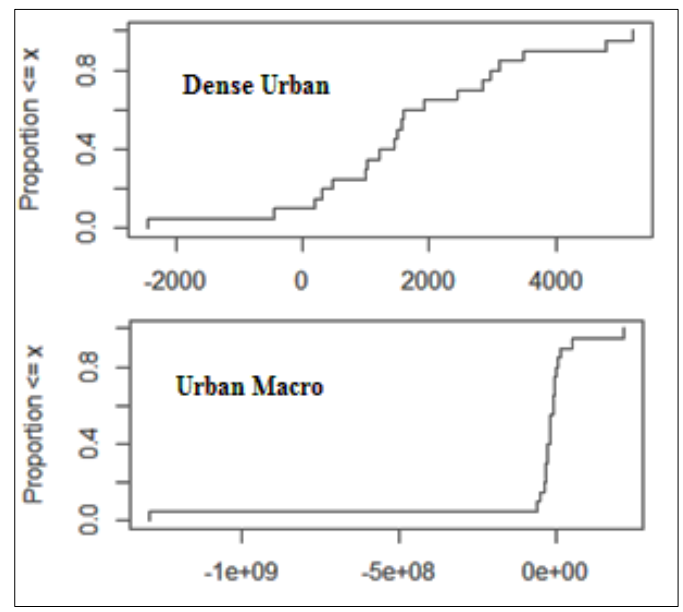

Figure 8. ECDF plot $(X=$ NPV)

We combine (1) - (11) in a single equation, in order to formulate the NPV as a function of cost and revenue decision variables. We treat each decision variable as belonging to a normal distribution, specifying its mean and standard deviation, to construct our range of input values. With the help of $\mathrm{R}$ simulations, we generate ECDF plot (Figure 8), to highlight the distribution of NPV values under the supplied input conditions [12]. We find that the chances of high returns (positive NPV) have larger probabilities $(\approx 0.7)$ for the case of Dense Urban scenario, as compared to the Urban
Macro Scenario $(\approx 0.3)$, for the specified range of input values of model parameters. Hence, operators may begin with the former deployment first.

We then evaluate the sensitivity of the NPV to the individual decision variables of the techno-economic model with the help of the partial rank correlation coefficient (PRCC) statistic [12]. PRCC evaluates the correlation of each decision variable with the NPV after removing the effect of all the other variables [12]. This furthers the prior work on sensitivity analysis [11] which incorporated only the TCO and the cost variables. We find that for both Dense Urban and Urban Macro scenarios, PRCC estimates are very similar. Table 7 ranks the model parameters in the order of their magnitude of impact on the NPV estimate. The sign in the brackets indicate the directionality of the relation.

Table 7. NPV decision variables

\begin{tabular}{|l|l|}
\hline High Impact & $\begin{array}{l}\text { Bandwidth (-), Spectrum Costs (-), } \\
\text { ARPU (+) }\end{array}$ \\
\hline $\begin{array}{l}\text { Moderate } \\
\text { Impact }\end{array}$ & $\begin{array}{l}\text { Cell range (+), BS Cost (-), WACC } \\
(-), \text { Loan rate (-), Savings rate (+) }\end{array}$ \\
\hline Low Impact & $\begin{array}{l}\text { Spectral Efficiency (+), Usage } \\
\text { Hours (-), Market Share (-) }\end{array}$ \\
\hline
\end{tabular}

\section{Conclusion}

This paper conducts a thorough techno-economic assessment for possible 5G HetNet deployment scenarios in the State of Texas, taking into account, for the first time, the advanced air interface technologies of Massive MIMO operating at the mmWave frequency of $28 \mathrm{GHz}$. Using Simple-Logistic forecast of 5G subscription for the 10-year horizon (20202030), we find that the Opex, Capex, and TCO requirements for such $5 \mathrm{G}$ configurations are almost one-third of that of an LTE-A configuration operating at $700 \mathrm{MHz}$ frequency. High positive returns with an NPV estimate of $\$ 482.14$ million should encourage the operators to go for $5 \mathrm{G}$ deployments. Higher probability $(\approx 0.7)$ of profits for the case of $5 \mathrm{G}$, compared to that of LTE-A $(\approx 0.3)$, should further bolster their confidence. The results in this study, thus, validate the postulates on the cost efficiency of Massive MIMO based 5G HetNets over the precursor technologies such as 4G LTE and LTE-A. However, for both 5G and LTE-A deployment scenarios, the managerial decision variables most sensitive to the NPV include: a) acquisition of spectrum - including when and how much to acquire, b) pricing of the services as reflected in the ARPU, and c) the choice of the carrier frequency which determines the cell range and the achievable coverage. 
Whether the LTE-A radio access technologies utilizing spectrum bands in the lower frequencies (sub $1 \mathrm{GHz}$ ) can positively influence the profitability aspects, if integrated intelligently with $5 \mathrm{G}$ HetNets having cellular layouts tuned to the demography of the deployment region, is the topic of our future work.

\section{References}

[1] W. Xiang, K. Zheng, and X. S. Shen, 5G mobile communications. Springer International Publishing, 2017.

[2] A. Gupta and R. K. Jha, "A Survey of 5G Network: Architecture and Emerging Technologies," IEEE Access, vol. 3, pp. 1206-1232, 2015.

[3] GSMA Intelligence, "Understanding 5G: Perspectives on future technological advancements in mobile," GSMA Report, 2014.

[4] V. Nikolikj, T. Janevski, and S. Member, "Profitability and Comparative Cost-Capacity Analysis of 5G Millimeter-Wave Systems," Proc. 22nd Telecomm. forum (TELFOR), 2014, vol. 7, pp. 256-259.

[5] A. Jha and D. Saha, "Techno-economic assessment of the potential for LTE based 4G mobile services in rural India," Proc. IEEE International Conference on Advanced Networks and Telecommuncations Systems, 2015, pp. 28-33.

[6] A. Jha and D. Saha, "Why is $700 \mathrm{MHz}$ band a Good Proposition for Provisioning pan-India 4G LTE Services ?," Proc. 9th International Conference on Communication Systems \& Networks, 2017, pp. 1-8.

[7] C. Bouras, P. Ntarzanos, and A. Papazois, "Cost Modeling for SDN / NFV Based Mobile 5G Networks," in Proc. 8th International Congress on Ultra Modern Telecommunications and Control Systems and Workshops (ICUMT), 2016, pp. 56-61.

[8] C. Bouras, V. Kokkinos, A. Kollia, and A. Papazois, "Techno-economic analysis of ultra-dense and DAS deployments in mobile 5G," Proc. Int. Symp. Wirel. Commun. Syst., vol. 2016-April, pp. 241-245, 2016.

[9] V. Nikolikj and T. Janevski, "Cost Modeling of Advanced Heterogeneous Wireless Networks under Excessive User Demand," in Mellouk A., Fowler S., Hoceini S., Daachi B. (eds) Wired/Wireless Internet Communications. Lecture Notes in Computer Science, vol 8458, Springer, Cham, 2014, pp. 68-81.

[10] G. Smail and J. Weijia, "Techno-economic Analysis and Prediction for the Deployment of 5G Mobile Network," Proc. 20th Conf. Innov. Clouds, Internet Networks, no. 2015, pp. 9-16, Mar. 2017.

[11] C. Bouras, A. Kollia, and A. Papazois, "Sensitivity Analysis of Small Cells and DAS Techno-economic Models in Mobile 5G," Proc. IEEE Wireless
Communications and Networking Conference, 2016, pp. 2742-2747.

[12] A. Chalom, C. Y. Mandai, and P. I. Prado, "Sensitivity analyses: a brief tutorial with $\mathrm{R}$ package pse." ver 0.3.1, pp. 1-14, 2013.

[13] “IDSER Demographic Data Analysis Tool.” [Online]. Available: http://idserportal.utsa.edu [Accessed: 12May-2017].

[14] "Verizon confirms XO spectrum in $28 \mathrm{GHz}$ and 39 $\mathrm{GHz}$ bands will be used in $5 \mathrm{G}$ tests." [Online]. Available: http://www.fiercewireless.com. [Accessed: 06-May-2017].

[15] A. Sulyman, A. Nassar, M. Samimi, G. Maccartney, T. Rappaport, and A. Alsanie, "Radio propagation path loss models for $5 \mathrm{G}$ cellular networks in the 28 GHZ and 38 GHZ millimeter-wave bands," IEEE Commun. Mag., vol. 52, no. 9, pp. 78-86, 2014.

[16] N. Meade and T. Islam, "Technological ForecastingModel Selection, Model Stability, and Combining Models," Manage. Sci., vol. 44, no. 8, pp. 1115-1130, Aug. 1998.

[17] S. F. Yunas, J. Niemela, M. Valkama, and T. Isotalo, "Techno-economical analysis and comparison of legacy and ultra-dense small cell networks," Proc. 39th Annu. IEEE Conf. Local Comput. Networks Work., pp. 768-776, 2014.

[18] C. Bouras, A. Kollia, and A. Papazois, "Dense Deployments and DAS in 5G: A Techno-Economic Comparison," Wirel. Pers. Commun., pp. 1-21, 2016.

[19] C. Bouras, V. Kokkinos, and A. Papazois, "Financing and Pricing Small Cells in Next-Generation Mobile Networks," in Mellouk A., Fowler S., Hoceini S., Daachi B. (eds). Lecture Notes in Computer Science, vol 8458, 2014, pp. 41-54.

[20] 5G-PPP, "5G PPP Architecture Working Group View on 5G Architecture." Working Paper for Public Consultation, 2016.

[21] BMI Database, "BMI Research.” [Online]. Available: www.bmiresearch.com/. [Accessed: 31-May-2017].

[22] G. Uwe, D. Northcott, P. Schuster, Investment Appraisal: Methods and Models. Springer Texts in Business and Economics, 2014.

[23] "Cost of Capital." [Online]. Available: http://pages.stern.nyu.edu/ adamodar/New_Home_Pa ge/datafile/wacc.htm. [Accessed: 16-May-2017].

[24] T. Bai, A. Alkhateeb, and R. Heath, "Coverage and capacity of millimeter-wave cellular networks," IEEE Commun. Mag., vol. 52, no. 9, pp. 70-77, 2014.

[25] "France sets $€ 2.5 \mathrm{~B}$ reserve price for $700 \mathrm{MHz}$ auction | FierceWireless." [Online]. Available: http://www.fiercewireless.com/europe/france-setseu2-5b-reserve-price-for-700-mhz-auction. [Accessed: 15-May-2017]. 